\title{
Correction to: Wnt/ $\beta$-catenin signaling mediates the suppressive effects of diallyl trisulfide on colorectal cancer stem cells
}

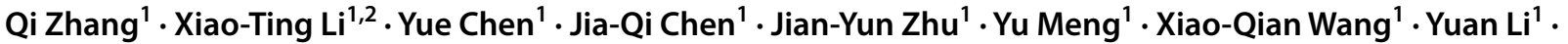 \\ Shan-Shan Geng ${ }^{1,2} \cdot$ Chun-Feng Xie ${ }^{1,2} \cdot$ Jie-Shu Wu ${ }^{1,2} \cdot$ Cai-Yun Zhong ${ }^{1,2}$ (D) Hong-Yu Han ${ }^{3}$
}

Published online: 30 April 2018

(c) Springer-Verlag GmbH Germany, part of Springer Nature 2018

\section{Correction to: Cancer Chemotherapy and Pharmacology https://doi.org/10.1007/s00280-018-3565-0}

Unfortunately, the online published article has error in

Fig. 4. The correct Fig. 4 is given here.

The original article can be found online at https://doi.org/10.1007/ s00280-018-3565-0.

Cai-Yun Zhong

cyzhong@njmu.edu.cn

$\bowtie$ Hong-Yu Han

hanhy@ sysucc.org.cn

1 Department of Nutrition and Food Safety, School of Public Health, Nanjing Medical University, 818 East Tianyuan Rd, Jiangning, Nanjing 211166, China

2 The Key Laboratory of Modern Toxicology, Ministry of Education, School of Public Health, Nanjing Medical University, Nanjing 211166, China

3 Department of Clinical Nutrition, Sun Yat-Sen University Cancer Center, State Key Laboratory of Oncology in South China, Collaborative Innovation Center for Cancer Medicine, Guangzhou 510060, China 
a

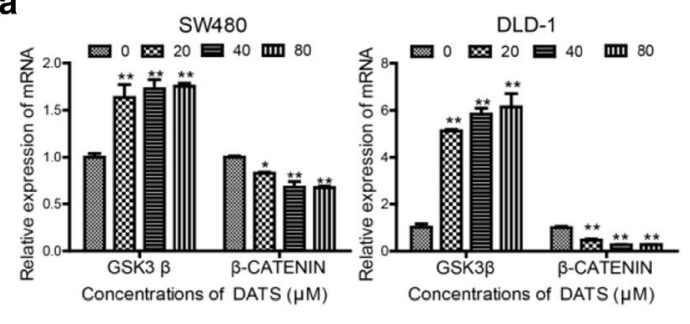

b

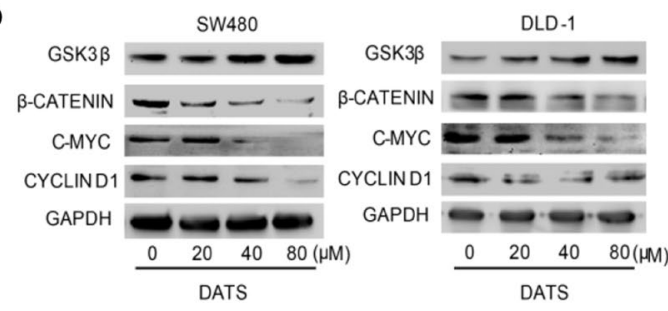

C

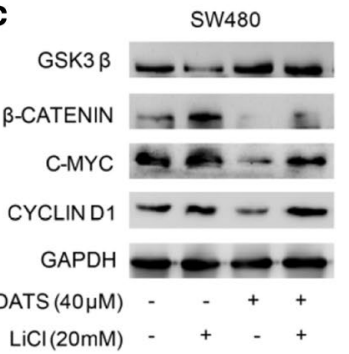
$\mathrm{LiCl}(20 \mathrm{mM})$ - + + +

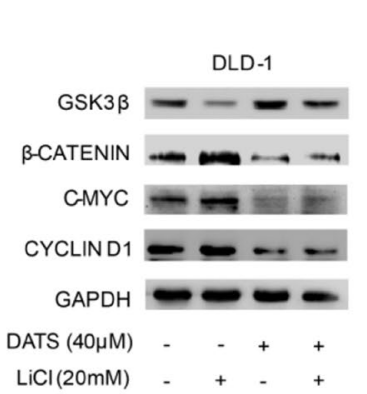

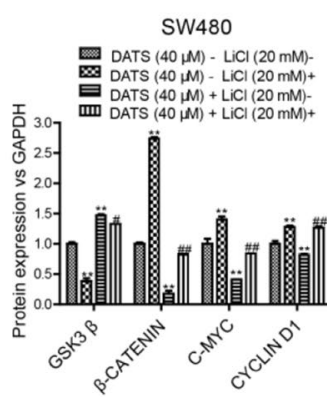

DLD-1

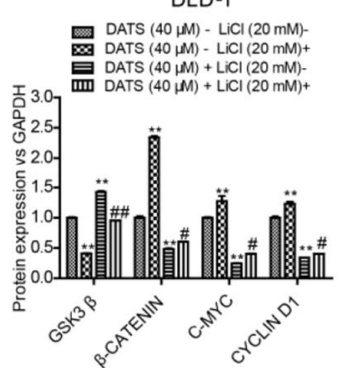

Fig. 4 DATS inhibites $W n t / \beta$-catenin pathway in colorectal CSCs. The tumorspheres enriched from SW480 and DLD-1 were treated with different concentrations of DATS for 5 days, a, b SW480 and DLD- 1 colonspheres were treated with DATS for 5 days. The expression levels of the critical molecules in $\mathrm{Wnt} / \beta$-catenin pathway were determined by qRT-PCR (a) and Western blotting (b), respectively. c-f SW480 and DLD-1 sphere-forming cells were cultured with $40 \mu \mathrm{M}$ DATS in the presence or absence of $20 \mathrm{mM} \mathrm{LiCl}$ for 5 days, and the protein levels for $\mathrm{Wnt} / \beta$-catenin pathway were detected by

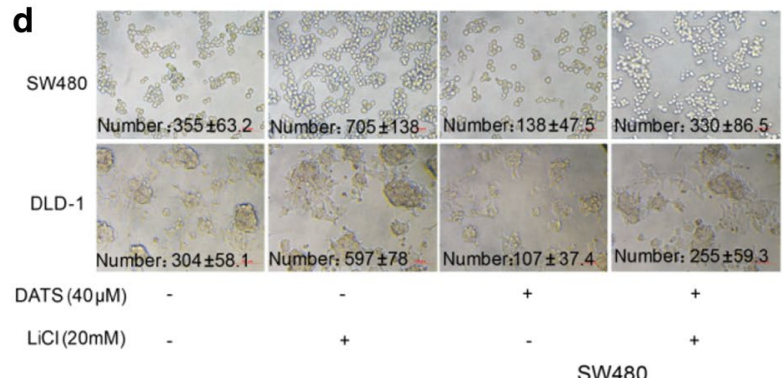

e
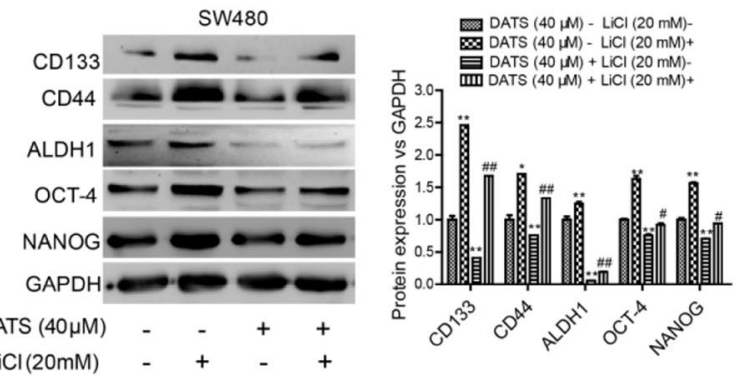

DLD-1

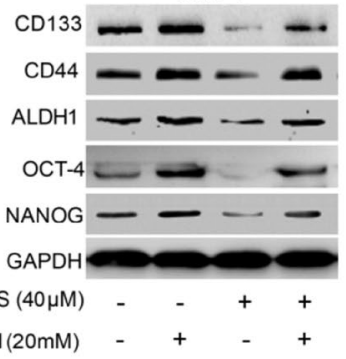

f

SW480
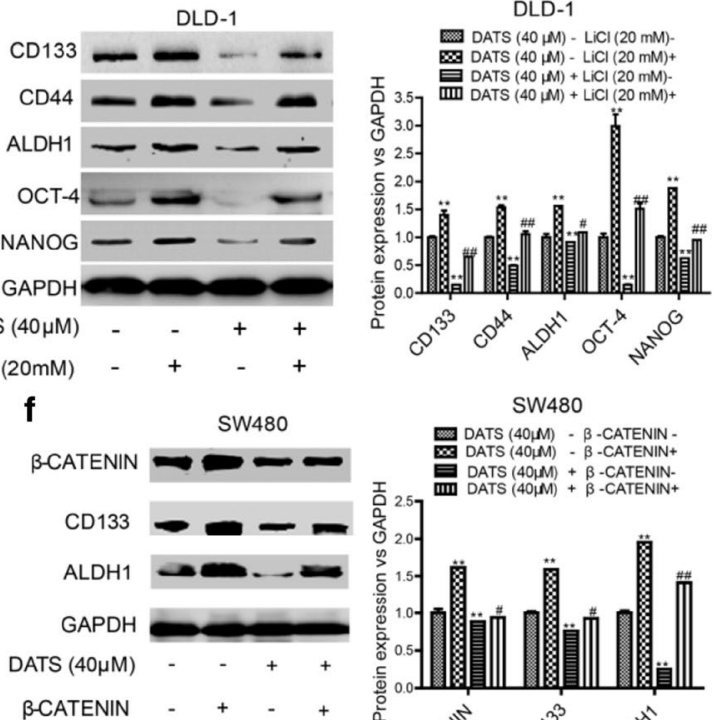

SW480

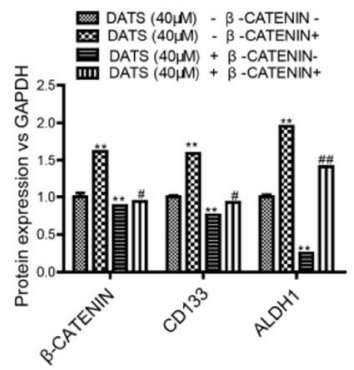

Western blotting (c). d The images of tumorspheres were obtained. Bar $100 \mu \mathrm{m}$. e Western blot analysis of colorectal CSCs markers. f SW480 cells were transfected with control vector or $\beta$-catenin plasmids, and then treated with or without DATS $(40 \mu \mathrm{M})$ for 3 days. The levels for $\beta$-CATENIN, CD133 and ALDH1 were detected by Western blotting. Data are expressed as mean $\pm \mathrm{SD}$ of three independent experiments. $* P<0.05$, $* * P<0.01$ compared with control group. ${ }^{\#} P<0.05,{ }^{\# \#} P<0.01$ compared with $40 \mu \mathrm{M}$ DATS group 